\title{
Reciprocal altruism in rats: Why does it occur?
}

\author{
Thomas R. Zentall ${ }^{1}$
}

Published online: 20 October 2015

(C) Psychonomic Society, Inc. 2015

Summary The finding of reciprocal altruism in rats does not explain how such behavior came to be. Attributing it to a genetic predisposition or basic learning processes may not be what the authors had in mind, but the alternative, cultural learning, seems even less plausible for rats and perhaps is not even the primary basis for reciprocal altruism in humans.

Many social species appear to show what Dawkins (1976) refers to as hard-core altruism. By providing resources for those who are related to us, that ability can be genetically selected for by means of kin selection (caring for our genes in others; Hamilton, 1964). Reciprocal altruism is a higher level of altruism because any animal that expends resources for another in the expectation of reciprocation risks the development in the gene pool of what Dawkins refers to as cheats, a genetic tendency to fail to reciprocate, and cheats will generally do better in terms of survival and reproduction than unconditional altruists (what Dawkins refers to as suckers). The way to counteract the advantage of cheats is to develop a tendency to be what Dawkins refers to as a grudger. A grudger will be altruistic initially, but if the other cheats and does not reciprocate, the grudger remembers the incident and bears a grudge. Thus, the key to the genetic advantage of reciprocal altruism is the ability to detect cheats. That means what is important for the development of grudgers is the capacity for individual recognition and good memory.

Thomas R. Zentall

zentall@uky.edu

1 Department of Psychology, University of Kentucky, Lexington, KY 40506, USA
What about the question of reciprocal altruism in nonhumans? According to this theory, good memory and individual recognition, at minimum, are required for reciprocal altruism. Thus, one might expect reciprocal altruism in species closely related to us, like apes and perhaps monkeys, but, surprisingly, Rutte and Taborsky (2008) found evidence for reciprocity in rats. Rats that had learned to pull a stick into their cage to gain access to food on a tray would also pull a stick that allowed another rat, called the focal rat, to gain access to food. Then, later, when the focal rat was allowed access to the stick that provided food for the rat in the next cage, it preferentially pulled the stick to feed the rat that had previously fed it rather than a control rat that had not fed it or a novel rat. This finding provided evidence for what the authors call direct reciprocity, involving recognition of the specific animal that fed them (what humans might call paying back). But they also found evidence for generalized reciprocity, the tendency to feed another rat even if the other rat was not the one that had provided them with food (what is sometimes referred to as paying it forward).

More recently, Dolivo and Taborsky (2015) found that a focal rat could not only identify the rat that fed it but could also distinguish the quality of food provided by the altruist. That is, the focal rat would preferentially feed a rat that had given it a preferred piece of banana rather than another that fed it a less preferred piece of carrot.

For a biologist, reciprocal altruism would be selected for if the cost of occasionally sharing one's resources with others was less than the benefit of receiving resources from others (at other times). Occasionally encountering an unknown cheat, who later would not reciprocate, adds to the cost. Presumably, those encounters are rare because those animals could be identified as cheats, and future altruism would not occur.

The approach of the experimental psychologist would be to ask what mechanisms might be responsible for this behavior. 
That is, how did this behavior come to be? More specifically, was this behavior genetically predisposed and released by an appropriate environmental context, or could it have been acquired by basic principles of reinforcement? Interestingly, when reciprocal altruism occurs in humans, it is often attributed to cultural factors, such as living by the golden rule, an acquired behavior. Reciprocal altruism in rats of the kind reported here would not be attributed to culture, but could it be explained in terms of learning?

In the research described by Rutte and Taborsky (2008), during pretraining, rats learned to pull a stick fixed to a baited platform to move it into its cage and obtain a reward. Thus, stick pulling was rewarded. In the second phase, each rat pulled the stick to provide a reward for another rat and had the stick pulled by the other rat for itself. At this point, it would appear to be altruistic, but could it be generalization from the first experience? That is, would these rats have pulled the stick even if the other cage had been empty, given that they had previously learned to pull a stick to receive food?

Still, in the actual experiment there was a tendency for the focal rat to preferentially pull the stick for a rat that had provided food rather than another rat on its other side that had not provided food. To explain this tendency, it is important to consider that the focal rats received food from one partner but not the other. That experience might result in a Pavlovian association between the food-providing rat and the food. That is, the focal rat was oriented to the food and so would have observed the provider when it was fed but not the nonprovider. Could that association have resulted in the focal rat facing the provider rather than the nonprovider when it had access to the stick? It is interesting that the authors note that each provider had been rewarded for pulling shortly before the focal rat was introduced to its compartment. Is it possible that the Pavlovian association of food in the presence of the provider, together with a generalized stick pulling response from the pretraining, provided the learning that was responsible for the apparent reciprocal altruism?

In the more recent experiment, Dolivo and Taborsky (2015) found that rats were more likely to pull the stick that fed a rat that had fed it a piece of banana than a different rat that had provided it with a piece of carrot. But given that the carrot was a less preferred reward, one might expect that the Pavlovian association with the carrot-providing rat would not be as strong as with the banana-providing rat. Thus, once again, when the focal rat had the opportunity to pull the stick, it would be more likely to orient to the rat that had provided it with the qualitatively better banana.

An important control procedure would be to test the focal rats when they were not deprived of food (I thank Jennifer Laude for this suggestion). If learning was responsible, the focal rat would be less likely to attempt to acquire the food. By contrast, if altruism was responsible for this behavior, it should make it even more likely to appear because one might expect altruism to be more probable when the focal rat doesn't need the resource.

What are the implications of this alternative account? I would not argue that a learning account is more credible than a genetic tendency to engage in reciprocal altruism based on group selection. On the other hand, it does raise an interesting question about similar examples of reciprocal altruism in humans.

When reciprocal altruism occurs in humans, we are likely to attribute it to cultural factors or learning. We are taught not only to "do unto others as you would have them do unto you" but also to show adequate appreciation for what others have done for us in the form of reciprocal altruism. This is our culture's way of encouraging us to go beyond what may be the instructions programmed into the selfish gene (Dawkins, 1976). But that is surely not why the rats are showing what appears to us as reciprocal altruism. If rats engage in this kind of behavior, it is likely to be either because that tendency is predisposed by their genes or, more likely, because the experiment happens to tap into associative processes that encourage it to emerge. But if a genetic predisposition or basic instrumental and Pavlovian processes are responsible for what appears to be reciprocal altruism in rats, could it also be responsible for similar behavior when it occurs in humans? Is it possible that cultural learning that encourages us to consider treating others fairly is simply our culture's way of reinforcing what would naturally occur either because our genes predispose us or learning that the behavior will be reinforced?

The finding of reciprocal altruism in rats does not explain how such behavior came to be. Attributing it to a genetic predisposition or learning may not be what the authors had in mind, but the alternative, cultural learning, seems even less plausible for rats and perhaps is not even the primary basis for reciprocal altruism in humans.

\section{References}

Dawkins, R. (1976). The selfish gene. Oxford: Oxford University Press.

Dolivo, V., \& Taborsky, M. (2015). Norway rats reciprocate help according to the quality of help they received. Biology Letters, 11(2). doi: 10.1098/rsbl.2014.0959

Hamilton, W. D. (1964). The genetical evolution of social behaviour. Journal of Theoretical Biology, 7, 1-16.

Rutte, C., \& Taborsky, M. (2008). The influence of social experience on cooperative behavior of rats (Rattus norvegicus): Direct vs, generalized reciprocity. Behavioral Ecology and Sociobiology, 62, 499505. 